\title{
Insulin production: from gene to granule
}

\author{
R . R egazzi ${ }^{1}$, C. B . Verchere ${ }^{2}$, P. A . H alban ${ }^{3}$, K . S. Polonsky ${ }^{4}$ \\ ${ }^{1}$ Institut de Biologie Cellulaire et de Morphologie, Lausanne, Switzerland \\ ${ }^{2}$ British Columbia Research Institute for Child and Family Health, Department of Pathology and Laboratory Medicine, \\ University of British Columbia, Vancouver, Canada \\ ${ }^{3}$ Laboratoires de Recherche Louis Jeantet, University of Geneva, Geneva, Switzerland \\ ${ }^{4}$ Department of Medicine, University of Chicago, Chicago, Illinois, USA
}

The pancreatic beta cell synthesizes and secretes appropriate amounts of insulin to allow correct regulation of blood glucose levels in non-diabetic individuals. In the short term, the beta cell releases precise quantities of insulin in response to minute-to-minute changes in blood glucose. In the long term, beta cells adapt to metabolic changes such as the insulin resistance associated with obesity by appropriately altering insulin synthesis and beta-cell mass. Defects in insulin production - from its synthesis through to its secretion - by the beta cell have been implicated in the pathophysiology of both insulin-dependent (IDDM) and non-insulin-dependent (NIDDM) diabetes mellitus. Recent investigations focussed on the biology of the beta cell have led to an explosion of new information on the pathways involved in insulin synthesis and secretion and how defects in these pathways might be implicated in the pathogenesis of diabetes. Thus, for example, during the past few years we have gained a further understanding of the regulation of insulin gene transcription and the development of normal differentiated beta-cell function; the important role of translational control in the regulation of proinsulin biosynthesis; the molecular physiology of the ion channels involved in the coupling of beta-cell glucose metabolism with insulin secretion; and the exocytotic machinery employed by beta cells to secrete insulin

Participants: M. A. Permutt, Washington University, St. Louis, Missouri, USA

M. S. German, University of California, San Francisco, California, USA

C. J. Rhodes*, University of Texas, Dallas, Texas, USA

F. M. Ashcroft, University of Oxford, UK

R. Regazzi, University of Lausanne, Switzerland

* unable to attend meeting

Corresponding author: P. A. Halban, Ph.D., Laboratoires de Recherche Louis Jeantet, 1, rue Michel Servet, CH-1211 Geneva 4 , Switzerland granules. Each of the genes encoding a protein involved in insulin production, from its biosynthesis to its release, represents a candidate susceptibility gene for diabetes; indeed, several recently identified genes associated with diabetes encode proteins that appear to play important roles in normal beta-cell function. Through improving our knowledge of the mechanism of insulin production by the beta cell, we will undoubtedly gain new insight into the pathogenesis of diabetes. We report here highlights from recent research devoted to the study of beta-cell biology.

Michael German: Transcriptional control of proinsulin biosynthesis and beta-cell differentiation

To fulfil their role as nutrient sensors and metabolic regulators, beta cells must express a set of specialized gene products, the most obvious being insulin. These genes start to be expressed as the beta cells develop from the intestinal epithelium and differentiate from the surrounding exocrine and endocrine cells. Both the process of development and the maintenance of the differentiated state are dependent on a unique set of transcription factor genes expressed in the beta cell.

It is important to understand that although they may activate transcription of beta-cell-specific genes, none of the beta-cell transcription factors are themselves expressed exclusively in beta cells. Instead the transcription factors have distinct but overlapping expression patterns that result in a set of factors that is absolutely unique to the beta cell. The transcription factors that compose this unique beta-cell set then interact to form unique functional complexes capable of activating beta-cell-specific genes such as insulin.

Michael German and his colleagues at the University of California, San Francisco, have used insulin gene transcription as a paradigm of beta-cell specific 
gene expression. The proximal (about $400 \mathrm{bp}$ ) insulin promoter is remarkably complex, with multiple sequence elements along its length. Because each of these sequence elements forms a recognition site for binding of beta-cell transcription factors, the promoter determines the structure of a transcriptional activation complex that can only be formed by the unique set of transcription factors found in the betacell nucleus.

Understanding the insulin gene, therefore, requires an understanding of the beta-cell transcription factors. The beta cells express multiple transcription factors, including many members of the basic helixloop-helix, winged helix, zinc finger, and homeodomain families of transcription factors. Among the homeodomain transcription factors expressed in beta cells are members of the hox, Lim, pou, Nkx, $\mathrm{cdx}, \mathrm{pdx}$ and paired families $[1,2]$.

As an example of a beta-cell transcription factor, Dr. German's laboratory has studied the expression and function of the paired homeodomain factor pax 6 . The pax family of vertebrate transcription factor genes is related to the Drosophila paired gene and shares a conserved sequence motif, the paired box, that encodes a DNA-binding domain. In addition to the pancreas, pax 6 is expressed in a variety of tissues, including the eye and the central nervous system. The importance of pax 6 in eye development has been demonstrated by the effect of mutations in D rosophila, rodents and humans. In mice, for example, mutation of pax 6 causes a syndrome termed small eye (Sey) because heterozygous animals have a reduction in eye size, while animals homozygous for the sey mutation have a complete disruption of eye development and severe craniofacial abnormalities and die at birth.

Dr. German and colleagues have observed that expression of pax 6 precedes that of the earliest pancreatic endocrine markers in the developing mouse embryo and remains restricted to cells of endocrine lineage throughout pancreatic development and in the adult pancreas. Homozygous small eye (Sey ${ }^{\text {neu }}$ ) mice have abnormal islet morphology and marked decreases in the numbers of all four islet cell types. In addition, islet morphology is disordered.

In the homozygous Sey ${ }^{\text {neu }}$ embryo, insulin and, more strikingly, glucagon production is severely attenuated due to decreased gene transcription. Pax 6 binds to crucial elements in the glucagon, insulin and somatostatin promoters; and pax 6, but not the Sey ${ }^{\text {neu }}$ (mutant pax 6) protein, activates insulin and glucagon transcription. These results demonstrate that pax 6 plays an important role both in islet development and in hormone gene transcription.

The remarkable effects of pax 6 on insulin gene expression underscore the importance of a complete set of transcription factors for full expression of the insulin gene. Despite the presence of multiple transcription factors in beta cells, the removal of a single factor can cripple the transcriptional activation complex. The understanding of both beta cell development and insulin gene transcription will require further studies of the expression and function of the beta-cell transcription factors. Such studies must include identification of the full set of beta-cell transcription factors, determination of their roles in development, and dissection of the interactions among transcription factors that produce the beta-cell transcription activation complexes.

\section{Christopher Rhodes: Translational control of proinsulin biosynthesis}

Following gene transcription, proinsulin biosynthesis is subject to further regulation at the level of translation. The importance of translational control in the normal regulation of proinsulin biosynthesis is becoming increasingly apparent from work performed by Christopher Rhodes and his colleagues at the University of Texas Southwestern Medical Center. In the short term $(<2 \mathrm{~h})$, glucose-stimulated proinsulin biosynthesis is regulated largely at the translational level. Thus, a 30-fold increase in proinsulin biosynthesis induced by glucose can be observed in just $1 \mathrm{~h}$, yet even after $6 \mathrm{~h}$ only a 2-3 fold glucose stimulation in preproinsulin mRNA levels is seen [3]. Since increased proinsulin biosynthesis places increased demand on proinsulin conversion, the beta cell compensates by a co-ordinated parallel glucose regulation of the biosynthesis of the prohormone convertase enzymes (PC1(3) and PC2), also at the translational level [4].

Although levels of preproinsulin mRNA do not change dramatically in response to glucose in the short term, there is a subcellular redistribution of preproinsulin mRNA following glucose stimulation. Dr. Rhodes has found that glucose rapidly decreases (within $30 \mathrm{~min}$ ) the cytosolic pool of preproinsulin mRNA while correspondingly increasing first the free polysomal associated preproinsulin mRNA, followed by an increase in the membrane-bound polysomal pool of preproinsulin mRNA (the major site of proinsulin biosynthesis in the beta cell). Glucose-induced ribosomal attachment to preproinsulin mRNA implies specific regulation at the initiation phase of translation.

Initiation of protein synthesis is known to be regulated by phosphorylation of eukaryotic initiation factors. However, no known eukaryotic initiation factors have yet been found to be phosphorylated in a way that correlates with the specific stimulation of proinsulin mRNA translation by glucose. Consequently, the presence of nutrient regulatory elements in the $5 '$ - and 3'-untranslated regions of preproinsulin mRNA has been proposed. While such elements 
have not yet been functionally identified, there are conserved regions in the untranslated regions of preproinsulin mRNA which are consistent with that hypothesis, including a 'stem loop' secondary structure in the 5 '-untranslated region and a highly conserved primary sequence in the 3 '-untranslated region, downstream of the polyadenylation signal.

The intracellular mediators coupling glucose metabolism in the beta cell to increased translation of preproinsulin mRNA also remain to be elucidated. Dr. Rhodes has outlined that none of the factors thought to be involved in coupling glucose metabolism to insulin secretion in the beta cell (e.g. ATP/ ADP, $\left[\mathrm{Ca}^{2+}\right]_{i}$; see later) appear to be involved. Preliminary data suggest that specific changes in the redox state of the beta cell, as reflected by a decreased cytosolic $\mathrm{NAD}(\mathrm{P}) \mathrm{H}: \mathrm{NAD}(\mathrm{P})$ ratio, may be involved.

As detailed in another report in this supplement, the important role of non-esterified fatty acids (NEFA) in the regulation of beta-cell function is becoming increasingly apparent. Interestingly, NEFA inhibit glucose-stimulated translation of preproinsulin mRNA, in marked contrast to the acute potentiation of glucose-induced insulin secretion by these agents. In isolated islets cultured in the presence of NEFA for $24 \mathrm{~h}$, insulin content decreases by $50 \%$ due to a fatty acid-induced increase in basal insulin secretion accompanied by a decrease in proinsulin mRNA translation. Thus, proinsulin biosynthesis fails to compensate for the fatty acid-induced insulin secretion. This finding may have implications for understanding the pathogenesis of beta-cell dysfunction in NIDDM, in which a role for NEFA has been implicated, and in which insulin secretion is increased but content is likely decreased. The decrease in insulin content induced by exposure of islets to NEFA occurs despite an increase in proinsulin gene expression, further emphasizing the importance of translation in the maintenance of insulin content.

\section{Frances Ashcroft: The role of ion channels in insulin} secretion

As reported by Frances Ashcroft of Oxford University, ATP-sensitive potassium channels $\left(\mathrm{K}_{\mathrm{ATP}}\right.$ channels) couple cell metabolism to electrical activity and play important roles in the physiology and pathophysiology of many tissues [5]. In pancreatic beta cells, the $\mathrm{K}_{\mathrm{ATP}}$ channel regulates insulin secretion both in response to glucose - the primary physiological stimulus - and to clinically important drugs. Mutations in this channel cause persistent hyperinsulinaemic hypoglycaemia of infancy (PHHI), a disease associated with unregulated hypersecretion of insulin. Defective regulation of $\mathrm{K}_{\mathrm{ATP}}$ channel activity, as a result of compromised beta-cell metabolism is associated with certain forms of maturity onset diabetes of the young (MODY).

The consensus view of beta-cell stimulus-secretion coupling is that when plasma glucose levels rise, glucose uptake and metabolism by the beta cell is increased. The resulting elevation of intracellular ATP, and concomitant lowering of intracellular MgADP, closes $\mathrm{K}_{\mathrm{ATP}}$ channels in the beta-cell plasma membrane, because ATP inhibits, whereas MgADP activates, channel activity. This produces a membrane depolarisation which activates voltage-dependent $\mathrm{Ca}^{2+}$ channels, increases $\mathrm{Ca}^{2+}$ influx into the beta cell and triggers insulin release. $\mathrm{K}_{\mathrm{ATP}}$ channels are also regulated by two clinically important classes of drugs. Sulphonylureas, widely used in the treatment of NIDDM, inhibit $\mathrm{K}_{\mathrm{ATP}}$ channel activity and thereby stimulate insulin release. By contrast, K-channel openers, such as diazoxide, activate $\mathrm{K}_{\text {ATP }}$ channels thereby hyperpolarizing the beta cell and inhibiting insulin release. Diazoxide is sometimes used to treat PHHI.

The beta-cell $\mathrm{K}_{\mathrm{ATP}}$ channel is a complex of two proteins: Kir6.2 and SUR1. Kir6.2 serves as the $\mathrm{K}_{\mathrm{ATP}}$ channel pore whereas the sulphonylurea receptor SUR1 acts as a regulator of channel activity conferring sensitivity to sulphonylureas and diazoxide. SUR1 belongs to a family of ATP-binding cassette transporters and, like other members of this family, has two large cytoplasmic domains which contain highly conserved consensus sequences for nucleotide binding. This suggests that the nucleotide binding domains (NBDs) of SUR1 may constitute one or more of the sites at which nucleotides regulate $\mathrm{K}_{\mathrm{ATP}}$ channel activity. Dr. Ashcroft and colleagues have therefore mutated (independently or together) two lysine residues (the $\mathrm{W}_{\mathrm{A}}$ lysines), one in the first (K719A) and one in the second (K1385M) NBDs of SUR1 [6]. These mutations are expected to abolish ATP hydrolysis without affecting nucleotide binding.

Their results indicate that neither $\mathrm{W}_{\mathrm{A}}$ lysine residue is required for channel inhibition by ATP: indeed, mutant currents were slightly more sensitive to ATP than wild-type currents. ADP has both stimulatory and inhibitory actions on wild-type $\mathrm{K}_{\mathrm{ATP}}$ currents (as demonstrated by the fact that MgADP activates, whereas free ADP inhibits, channel activity). Dr. Ashcroft's group has further shown that the ability of MgADP to potentiate $\mathrm{K}_{\mathrm{ATP}}$ currents required the presence of the $\mathrm{W}_{\mathrm{A}}$ lysine in both NBDs. Inhibition of $\mathrm{K}_{\mathrm{ATP}}$ currents by ADP, like that of ATP, did not require the $\mathrm{W}_{\mathrm{A}}$ lysines. The guanine nucleotides, MgGDP and MgGTP, also stimulated wild-type but not $\mathrm{W}_{\mathrm{A}}$ mutant currents. Although their data argue that the $\mathrm{W}_{\mathrm{A}}$ lysine residues are required for MgADP (or MgGDP) binding to produce a conformational change which leads to channel activation, they do not allow a conclusion as to whether or not this conformational change requires nucleotide diphosphate hydrolysis. 
$\mathrm{K}_{\mathrm{ATP}}$ channel regulation by $\mathrm{MgADP}$ plays a central role in coupling changes in beta-cell metabolism to channel activity. Recent results indicate that this regulation is achieved by the NBDs of SUR1, which in some way influence the opening of the Kir6.2 pore. Metabolic inhibition led to activation of wildtype but not K719A or K719A/K1385M currents, supporting the idea that $\mathrm{MgADP}$ plays an essential role in metabolic regulation of the $\mathrm{K}_{\mathrm{ATP}}$ channel.

The stimulatory effect of diazoxide requires the presence of hydrolysable nucleotide at the inner membrane surface. Dr. Ashcroft's results indicate that the $\mathrm{W}_{\mathrm{A}}$ lysine at NBD1 is essential for activation of $\mathrm{K}_{\mathrm{ATP}}$ currents by diazoxide, while that at NBD2 has an enhancing role. This suggests that a conformational change induced by nucleotide binding or hydrolysis at NBD1 is involved in diazoxide action. Dr. Ashcroft also found that $\mathrm{MgGDP}$ was more effective at supporting diazoxide action than $\mathrm{MgGTP}$, which argues that interaction of nucleotide diphosphates with the NBDs of SUR1 induces this conformational change more readily than nucleotide triphosphates.

These studies clarify the mechanism of action of nucleotides on $\mathrm{K}_{\mathrm{ATP}}$ channels and demonstrate that the $\mathrm{W}_{\mathrm{A}}$ lysines within the NBDs of SUR1 play an essential role in channel activation by $\mathrm{MgADP}$, MgGDP and diazoxide.

\section{Romano Regazzi: Molecular mechanism of insulin exocytosis}

The fine tuning of insulin secretion from pancreatic beta cells is essential for blood glucose homeostasis. Molecular dissection of the exocytotic event is a prerequisite to understand how secretagogues such as glucose stimulate insulin release and how such regulation becomes deranged in disease states. As reported by Romano Regazzi of the University of Lausanne, although much remains to be done until the detailed mechanism underlying insulin secretion is elucidated at the molecular level, studies performed during the last couple of years have brought an extraordinary development in our knowledge about the basic components of the exocytotic machinery in pancreatic beta cells.

Advances in different research fields, including reconstitution of vesicular trafficking in cell-free systems, studies on the mode of action of clostridial neurotoxins and genetic studies in yeast have led to the formulation of a general model to explain how secretory vesicles in eukaryotes are targeted and fuse with the appropriate acceptor membrane [7]. This is a fundamental issue since secretory products such as insulin travel in a highly regulated fashion from the endoplasmic reticulum to the Golgi apparatus and from there toward the plasma membrane. According to this model each secretory vesicle possesses at least one protein that functions as a vesicular SNAP receptor (v-SNARE). The v-SNARE interacts specifically with protein(s) located on the targeted membrane, the target SNAP receptor(s) (t-SNARE). The complex formed between v- and t-SNAREs serves two functions: first, the specific pairing between proteins located on the secretory vesicle and on the acceptor membrane ensures correct targeting of the vesicle; second, the SNARE complex creates a kind of scaffold that permits the recruitment of cytosolic components required for vesicle fusion. During the last 2 years both the v-SNAREs of insulin-containing secretory granules and the t-SNAREs located at the plasma membrane of pancreatic beta cells have been identified [7]. Insulin-secretory granules carry two vSNAREs belonging to the VAMP/synaptobrevin family, VAMP-2 and cellubrevin, while SNAP-25 and syntaxin-I are associated with the plasma membrane [7]. The involvement of these proteins in insulin exocytosis was demonstrated using the neurotoxins produced by Clostridium tetani (TeTx) and Clostridium botulinum (BoNT). These neurotoxins are zinc-dependent proteases with a unique substrate specificity. Thus, BoNT serotype B, D and F cleave VAMP proteins (both VAMP-2 and cellubrevin), BoNT A and E cut SNAP-25 while BoNT C cleaves syntaxin-I. The introduction of each of these neurotoxins in permeabilized cells (pancreatic beta cells do not express the neurospecific receptors for the toxins) results in a block of $\mathrm{Ca}^{2+}$-triggered exocytosis clearly demonstrating that VAMPs, SNAP-25 and syntaxin-I are required for insulin secretion [7]. Secretory granules of pancreatic beta cells possess two v-SNAREs that are cleaved equally well by clostridial neurotoxins. Thus, using this approach it was not possible to assess the implication of each of the two VAMP isoforms in insulin exocytosis. For this purpose a different strategy was designed by Regazzi and colleagues to analyse the respective roles of VAMP-2 and cellubrevin in the control of insulin release [8]. Each of the two isoforms was made resistant to TeTx proteolysis by modifying the amino acids at the cleavage site and was then transfected in insulin-secreting cells. The transfected cells were permeabilized and treated with TeTx to inactivate the endogenous VAMPs but not their genetically engineered counterparts whose cleavage site for the toxin was modified. Using this technique VAMP-2 and cellubrevin were found to reconstitute equally well $\mathrm{Ca}^{2+}$-evoked secretion in TeTx-treated cells indicating that both VAMPs are implicated in the control of insulin exocytosis [8]. Deletion mutations of VAMP proteins preventing the binding to SNAP-25 and/or syntaxin-I rendered the proteins inactive, confirming that the interaction between $v$ - and t-SNAREs is an essential requirement for the exocytotic process [8].

Ras-like GTPases of the Rab family are implicated in vesicular trafficking and are required for each step 
along the secretory pathway [7]. The precise mode of action of Rab proteins is not yet fully understood but these GTPases are believed to contribute to the specificity of vesicle targeting. A current model proposes that Rab proteins favour or stabilize the interaction between $\mathrm{v}$ - and t-SNAREs enabling secretory vesicles to reach their proper destination. Pancreatic beta cells express several members of the Rab family associated with different cellular compartments. In particular, different Rab3 isoforms are associated with insulin-containing secretory granules [7]. To demonstrate a functional role of Rab3 proteins in the regulation of insulin secretion, HIT-T15 were transfected with wild-type Rab3A and with a GTPase deficient mutant of this Rab protein. Overexpression of wild-type Rab3A did not affect basal secretion but resulted in a small inhibition of nutrient-evoked insulin release. This inhibition was much more pronounced in cells transfected with the GTPase deficient mutant of Rab3A. Thus, GTP hydrolysis by Rab3A is essential for the exocytotic process of beta cells.

The investigations performed during the last couple of years have thus led to the identification of several proteins required for the targeting and fusion of insulin-containing secretory granules with the plasma membrane. The next challenge will be to understand how physiological stimuli are able to modulate the exocytotic machinery of pancreatic beta cells.

\section{Alan Permutt: Beta-cell genes in diabetes}

Genes encoding beta-cell proteins are attractive candidates for susceptibility genes for both IDDM and NIDDM. This seems especially true for NIDDM, as there is considerable evidence to suggest that NIDDM is a polygenic disease in which genetic defects in beta-cell function are present. M. Alan Permutt and his colleagues at Washington University in St. Louis are using a molecular genetic approach to uncover the aetiology of NIDDM by investigating the hypothesis that while insulin resistance is a major component of the disease, those individuals with defects in insulin production are at greatest risk. Such studies typically utilize a number of genetic methods which are integrated with the development of the $\mathrm{Hu}$ man Genome Project. One such approach is to use human islet cDNA libraries to identify novel islet genes [9]. In recent years, these libraries have been used to isolate a number of genes involved in insulin biosynthesis and secretion, including the rate-limiting proteins for islet glucose metabolism, the islet glucose transporter GLUT2 and the islet glucose phosphorylating enzyme glucokinase. Other islet cDNAs isolated and characterized include mitochondrial glycerolphosphate-dehydrogenase (mGPDH), the sulfonylurea receptor (SUR), the ATP-regulated potassium channel Kir6.2, and several other unique islet potassium channel genes including Girk 1, Girk 2, Cir, and a calcium-activated potassium channel hSlo.

Once the cDNA for a beta-cell gene has been isolated, several approaches can be used to determine whether this represents a diabetes gene. For example, one can identify genomic clones with nearby microsatellite markers and place the genes on physical maps using radiation hybrid and yeast artificial chromosome (YAC) contig mapping, then look for linkage in families or screen known diabetic loci. In addition, a number of beta-cell genes have now been screened by Dr. Permutt and others for single nucleotide changes in diabetic patients by single-strand conformational polymorphic analysis (SSCP) and direct genomic sequencing. Several mutations have been found in the glucokinase gene which are responsible for a large fraction of MODY, a relatively rare form of NIDDM. A single mutation was found in the islet glucose transporter which completely eliminated glucose entry, defining a unique portion of the protein required for glucose transport. Variants of mGPDH, SUR [10], and $\mathrm{K}_{\mathrm{ATP}}$ channels were also identified, and SUR variants were found to be significantly associated with NIDDM.

A number of islet transcription factors have recently been identified which appear to be important in regulation of insulin biosynthesis. Dr. Permutt's group has isolated human clones of the genes encoding these transcription factors, identified genomic structure and mapped their chromosomal localization, and they are currently in the process of screening diabetic patients for mutations in these genes. Islet transcription factors such as Nkx 6.1/Nkx 6A, Isl1, and Pdx 1 represent good candidates for NIDDM genes, since this disease is characterized by a failure to increase beta-cell mass and insulin production in the presence of insulin resistance. In this regard, it is interesting to note that mutations in hepatic nuclear factor (HNF)1 $\alpha$, a transcription factor expressed in islets, have been identified in patients with MODY3, a group who are known to have impaired insulin secretion. Exon 4 of the HNF1 $\alpha$ gene appears to contain a mutational hotspot, as 30-40 different mutations have recently been found in this region of the gene. Whether the HNF1 $\alpha$ gene or indeed genes encoding other islet transcription factors play a role in more common forms of NIDDM remains to be determined; Dr. Permutt has now sequenced the HNF1 $\alpha$ gene in 24 diabetic Ashkenazi Jews, and found a nonsense mutation in one individual.

An additional strategy for identification of novel islet genes has been the use of differential display of islet mRNA. A major limitation of islet cDNA libraries is the presence of contamination by cDNAs encoding proteins expressed in exocrine pancreas. To circumvent this problem, Permutt and colleagues are using differential display to identify, clone, and sequence genes uniquely expressed in islet mRNA 
relative to pancreatic exocrine tissue. These islet expressed sequence tags (ESTs) have been compared to sequences in public databases. In several cases, interesting previously known genes such as the calcium-activated potassium channel and a DNA helicase have been identified, as well as a new sulfotransferase which might play a role in sex steroid/glucocorticoid metabolism in islets. In addition, they have identified over 40 novel islet ESTs which have been placed in the human genetic map. These novel islet ESTs will be useful for positional cloning of NIDDM genes.

Positional cloning represents an additional approach to identifying diabetes genes that is useful in monogenic disorders. Using positional cloning, Dr. Permutt's group successfully mapped a monogenic disorder of insulin secretion, that of Familial Hyperinsulinism. Children with this condition are born with severe hypoglycaemia and uncontrolled insulin secretion. After performing a whole genome search with anonymous markers and mapping the disease to the short arm of chromosome 11, a candidate gene, the sulfonylurea receptor, was mapped to this region. This gene was subsequently shown to be a regulatory component of the $\mathrm{K}_{\mathrm{ATP}}$ channel. Dr. Permutt has now characterized the SUR gene and the Kir6.2 gene in approximately 100 probands with Familial Hypoglycaemia, and has identified 19 mutations in these genes (unpublished data), including one child with a homozygous nonsense mutation in the $\mathrm{K}_{\mathrm{ATP}}$ channel. This child presents the first functional knockout of the Kir6.2 gene found in man.

\section{Conclusion}

Thanks to basic research on the biology of the beta cell, we have moved much closer to a complete understanding of the fundamental mechanisms involved in insulin synthesis and secretion. Such information will be critical to the future development of any novel therapies for the treatment of diabetes. Indeed, it is only after having a clear picture of how the normal beta-cell functions that we will be able to devise effective pharmacological agents which act on the diabetic beta cell or to bioengineer surrogate beta cells. Through this work we have also furthered our understanding of how mutations in beta-cell proteins may lead to diabetes and have identified novel proteins involved in insulin production, some of which represent excellent new candidate genes for diabetes.

Despite the remarkable progress made over the past few years, it is clear that much more work is needed before the most intimate mysteries of the beta cell are revealed and exploited for therapeutic purposes. Future work in the areas of research discussed above and with a view to improved treatment and/or prevention of diabetes are envisaged to include:

\section{Future directions and recommendations}

- Increase our basic understanding of the promoter elements and transcription factors involved in insulin gene transcription and beta-cell differentiation and how these elements may be involved in the beta-cell adaptation to insulin resistance.

- Define the mechanism of stimulus-translation coupling by which nutrient metabolism regulates translation of proinsulin and other mRNAs in the beta cell.

- Identify the ion channels that maintain the resting membrane potential and the bursting activity of beta cells.

- Elucidate how calcium and other factors interact with SNARE proteins in order to stimulate exocytosis of insulin granules.

- Identify novel beta-cell genes involved in diabetes. - Establish a catalogue of "minimal essential" proteins indispensable for achieving regulated insulin secretion and necessary for the creation of surrogate beta cells.

\section{References}

1. Kennedy G, German M (1996) Insulin gene regulation. In: LeRoith D, Olefsky J, Taylor S (eds) Diabetes mellitus: a fundamental and clinical text. Lippincott-Raven, Philadelphia, pp 20-26

2. Sander M, German MS (1997) The $\beta$-cell transcription factors and development of the pancreas. J Mol Med (in press)

3. Schuppin GT, Rhodes CJ (1996) Specific co-ordinated regulation of PC3 and PC2 gene expression with that of preproinsulin in insulin-producing beta TC3 cells. Biochem $\mathbf{J}$ 313: $259-268$

4. Alarc'on C, Lincoln B, Rhodes CJ (1993) The biosynthesis of the subtilisin-related proprotein convertase PC3, but not that of the PC2 convertase, is regulated by glucose in parallel to proinsulin biosynthesis in rat pancreatic islets. $\mathbf{J}$ Biol Chem 268: 4276-4280

5. Ashcroft FM, Rorsman P (1990) Electrophysiology of the pancreatic $\beta$-cell. Prog Biophys Mol Biol 54: 87-143

6. Gribble FM, Tucker, SJ, Ashcroft FM (1997)The essential role of the Walker A motifs of SUR1 in K-ATP channel activation by MgADP and diazoxide. EMBO J 16: 1145-1152

7. Wollheim CB, Lang J, Regazzi R (1996) The exocytotic process of insulin secretion and its regulation by $\mathrm{Ca}^{2+}$ and G-proteins. Diabetes Rev 4: 276-297

8. Regazzi R, Sadoul K, Meda P, Kelly RB, Halban PA, Wollheim CB (1996) Mutational analysis of VAMP domains implicated in $\mathrm{Ca}^{2+}$-induced insulin exocytosis. EMBO J 15: 6951-6959

9. Ferrer J, Wasson J, Schoor KP, Mueckler M, Donis-Keller H, Permutt MA (1997) Mapping novel pancreatic islet genes to human chromosomes. Diabetes 46: 386-392

10. Nestorowicz A, Wilson BA, Schoor KP et al. (1996) Mutations in the sulfonylurea receptor gene are associated with familial hyperinsulinism in Ashkenazi Jews. Hum Molec Genet 5: 1813-1822 